\title{
Marketing senior et résistances des jeunes retraités au vieillissement
}

\author{
Hélène Eraly \\ Doctorante en sociologie, Université de Namur \\ Laboratoire d'anthropologie prospective \\ Université catholique de Louvain (Belgique)
}

\begin{abstract}
[Résumé] Depuis quelques décennies, le marketing senior s'est développé se faisant le relais des normes dominantes en matière de vieillissement. Les salons pour seniors sont un terrain privilégié d'étude de ces normes. En effet, ceux-ci enjoignent à résister au vieillissement individuel. Cette injonction à la résistance trouve un écho favorable chez les jeunes retraités qui opposent une résistance aux contraintes de l'avancée en âge par la recherche d'épanouissement personnel et l'action individuelle. Mais ces valeurs ne sont pas partagées par tous, de sorte que l'on ne peut affirmer qu'il n'existe qu'une seule manière de vivre son vieillissement à la retraite.

Mots-clés : retraite, vieillissement, marketing senior, épanouissement, action personnelle.
\end{abstract}

[Abstract] The senior marketing has developed in recent decades. He became the relay of the dominating aging norms. The "seniors' exhibitions" are a special field of survey to study these norms. These exhibitions enjoin a resistance to individual aging. This injunction receives a favorable response among young retirees. Indeed, they resist the constraints of advanced age by seeking self-realization and individual action. But these values are not shared by all, so we cannot say that there is only one way to experience aging at retirement.

Keywords: retirement, aging, senior marketing, self-realization, individual action.

T e dernier tiers du XXe siècle a connu un mouvement de généralisation des exiLgences de réalisation de soi et de responsabilité individuelle (Giddens 1991; Martuccelli 2002 ; Honneth 2006). Si l'individu moderne est responsable de trouver une manière de se réaliser dans les différentes épreuves de sa vie, le passage à la retraite n'échappe pas à cette norme. En effet, avec l'augmentation de l'espérance de vie, le développement des politiques de préretraites durant les années 1980 et 1990, et l'amélioration générale des conditions matérielles, les retraités disposent aujourd'hui de temps et de moyens financiers bien supérieurs à ceux de leurs prédécesseurs (Gilleard et Higgs 2011). Dans cette perspective, la retraite est généralement vécue comme une nouvelle phase de la vie dans laquelle l'individu, enfin libéré des contraintes professionnelles, peut se réaliser et profiter du temps qui lui reste.

Le marketing a bien intégré ces transformations sociales et a progressivement dessiné une nouvelle figure du consommateur, celle du « senior» (Caradec 2005). Il a contribué à développer le modèle de la «retraite-épanouissement »(Gestin 2001) ; la re- 
traite est devenue un temps où l'on profite de sa nouvelle liberté. Le marketing a aussi participé à la transformation de l'image de la vieillesse sur laquelle chacun peut/doit désormais avoir une maîtrise en s'occupant de son corps et de son esprit. À travers ces normes, il participe à la diffusion d'un vieillissement dit « actif ».

Le marketing senior constitue une porte d'entrée particulièrement pertinente pour étudier la diffusion des normes de responsabilisation et d'épanouissement, bien qu'il ne soit pas le seul. Ainsi, plusieurs recherches ont montré que le discours du « vieillissement actif » était également porteur de ces normes, que ce soit dans le domaine de la santé publique (Hénaff-Pineau 2009), des politiques d'emploi (Moulaert et Léonard 2011; Walker 2009) ou dans l'espace médiatique (Gestin 2003).

Le marketing propose des modèles de consommation qui s'inspirent des pratiques individuelles et condensent des normes sociales. Pour cette raison, la sociologie ne peut ignorer le marketing senior qui se développe depuis quelques années.

Une étude sociologique du marketing implique d'appréhender celui-ci dans sa dimension communicationnelle: quels messages sont véhiculés par le marketing ? Comment sont-ils transmis et à qui ? C'est ce que nous aborderons dans la première partie en prenant pour terrain spécifique les salons pour seniors. Nous analyserons le type de messages et d'images qui sont véhiculés dans ces salons afin de comprendre comment ceux-ci peuvent participer à la reproduction de modèles partagés. Nous verrons que tout en valorisant le troisième âge, ces salons enjoignent à une résistance aux signes de la « vieillesse-dépendance ».

Dans la seconde partie, nous répondrons à la question: comment ces messages sont-ils reçus et interprétés ? Éric Dacheux postule que « toute communication [...] nécessite un sens commun que l'on considère comme admis et que l'on ne renégocie pas » (Dacheux 2001: 418). C'est ce «sens commun» coproduit par les différents acteurs que nous souhaitons interroger dans cet article. Nous chercherons à comprendre l'incidence qu'ont ces injonctions au « vieillir jeune » sur les pratiques quotidiennes des retraités. Nous partirons de l'hypothèse que ces injonctions sont des modèles normatifs desquels chacun s'inspire pour orienter et juger sa propre conduite (Hewitt 2010; Ricoeur 1990). Nous analyserons donc les traces de ces cadres normatifs dans les pratiques des jeunes retraités à partir des récits qu'ils en font en mettant en évidence les résistances opérées par les individus. Nous distinguerons deux formes de résistance : les résistances à des contraintes physiques, lorsque les retraités luttent contre le vieillissement corporel et la « vieillesse-dépendance»; et les résistances à des contraintes morales, lorsqu'ils cherchent à se distinguer des représentations de la retraite et de la vieillesse. Enfin, pour éviter une généralisation abusive, et pour rester les plus fidèles possibles au corpus d'entretiens, nous évoquerons le cas de deux ouvriers qui ne partagent pas le même cadre de valeurs et qui font ainsi exception aux analyses apportées. 


\section{Dispositif méthodologique}

Les analyses proposées s'appuient sur une recherche doctorale par entretiens qualitatifs menée depuis plus de deux ans sur les reconfigurations identitaires au moment du passage à la retraite et sur le rôle joué par la consommation dans ces processus. Pour étudier la retraite dans sa dimension consumériste, nous avons choisi d'observer les Salons pour seniors de Paris et de Bruxelles.

$\mathrm{Au}$ total, 32 entretiens approfondis ont été menés avec 15 hommes et 17 femmes belges âgés de 55 à 68 ans. Afin de fournir un panorama le plus large possible des vécus différenciés de cette transition, nous avons cherché à diversifier au maximum notre échantillon : les personnes interrogées sont issues de milieux socioéconomiques diversifiés :

- cadres et professions intellectuelles supérieures : 4 managers, 1 orthodontiste, 1 fonctionnaire européen, 2 psychologues, 1 ingénieur, 1 analyste financière ;

- professions intermédiaires : 3 instituteurs, 1 directeur d'école, 1 assistant social, 3 infirmiers ;

- employés : 1 policier, 1 puéricultrice, 1 comptable, 8 employés administratifs ;

- ouvriers : 1 conducteur de bus, 1 balayeur de rue, 1 technicienne de surface.

\section{Le marketing senior comme (re)producteur de normes sociales}

L'analyse sociologique du marketing ne peut se réduire à concevoir celui-ci comme une méthode purement rationnelle visant à produire un maximum de demandes pour un produit donné, comme le fait la gestion. Elle ne peut non plus s'inscrire uniquement dans une visée normative critique postulant qu'il ne s'agit que d'un outil de persuasion et de manipulation des masses (Dacheux 2001). Autrement plus complexe, le marketing cristallise à travers les produits qu'il propose et les messages qu'il envoie, plusieurs phénomènes : il relaye les discours dominants (politiques, médicaux, etc.) et s'inspire des "styles de vie» (Giddens 1991) des consommateurs (leurs goûts, leurs aspirations, leurs manières de voir le monde), pour produire des imaginaires ou recréer des stéréotypes autour d'un produit et d'une réalité sociale (Desjeux 2010). Nous pouvons dire, dans la foulée de l'approche critique de la consommation, que l'objectif même du marketing est de tenter, par un ensemble d'artifices, de masquer la consommation au sens strictement marchand du terme, et de recréer un semblant de réalité dans laquelle le consommateur est invité à se projeter (Baudrillard 1970).

Le marketing senior s'est développé depuis les années 1980 afin d'ouvrir de nouveaux marchés, plus adaptés aux transformations du vieillissement. Les personnes âgées ont acquis une place dans les représentations publicitaires, et leur image s'est transformée : à la figure du grand-parent âgé dépourvu de mobilité et s'occupant de sa famille (Antoine 2003) s'est substituée une représentation "enchantée » de la vieillesse (Ruaux 2007) qui privilégie le jeune retraité disposant de temps, de moyens fi- 
nanciers et de capacités physiques, et qui investit sa retraite comme un moment d'épanouissement personnel. Le marketing a ainsi participé à créer la figure aujourd'hui bien connue du « senior » (Caradec 2005), tournée vers un futur qu'elle prépare, et cherchant à se réaliser (Bradley et al. 2001).

Agathe Gestin a montré que c'est le modèle de la « retraite-épanouissement » (Gestin 2001) qui s'impose aujourd'hui dans le champ de la consommation. Celui-ci repose sur l'importance du "vieillir-jeune » : réussir sa retraite c'est s'épanouir en restant jeune et en forme, en multipliant les loisirs, en s'impliquant dans sa famille et dans la société. La clé de cet épanouissement n'est autre que l'action individuelle (par le sport, la nourriture, etc.). Autrement dit, le marketing mobilise dans les messages qu'il diffuse les exigences de la modernité que sont la responsabilité individuelle ("soyez prévoyants », " restez jeunes ») et la réalisation de soi ("investissez des activités que vous valorisez »). La « vieillesse-dépendance » demeure dès lors majoritairement - mais pas totalement, nous le verrons - exclue du paysage publicitaire.

Cette image de la personne âgée se déploie à travers plusieurs canaux. Il y a les formes classiques de publicité telles que les encarts dans des magazines ou les spots télévisés représentant des personnes grisonnantes en pleine activité sportive, sociale ou familiale (Feillet et al. 2010). Une presse spécialisée s'est également développée, proposant des magazines conçus spécifiquement pour les personnes âgées de plus de cinquante ans, prodiguant des conseils financiers, psychologiques, juridiques, médicaux et affectifs (Gestin 2003). Ont également émergé les salons pour seniors, des espaces qui concentrent tous ces supports médiatiques à la fois et qui sont, à ce titre, un lieu d’observation privilégié pour notre objet.

\section{Les salons pour seniors, espaces de cristallisation du marketing senior}

Les salons commerciaux rassemblent dans un seul lieu des exposants et des visiteurs autour d'un thème partagé dans un objectif commercial (Cousin 2005). Le salon ne juxtapose pas simplement des catégories de marchandises (comme dans le supermarché), il les «culturalise » (Baudrillard 1970 : 21). C'est-à-dire qu'il concentre dans un même espace des objets de consommation de natures bien différentes avec leurs contextes de consommation propres, en proposant au consommateur une interprétation, un imaginaire d'utilisation de ces objets. Sont ainsi exposés dans les salons de seniors différents types d'espaces comme un salon de beauté, une salle de fitness, une cave de dégustation de vin, etc., autant de lieux recréés artificiellement dans le but d'éveiller l'imaginaire consumériste des visiteurs afin qu'ils s'y projettent.

Les salons pour seniors ont cette particularité de présenter deux faces du vieillissement, à défaut d'en montrer davantage: le "vieillir-jeune» et la «vieillessedépendance ». La majorité des exposants présents dans ces salons commercialisent des objets et des services liés au « vieillir-jeune ». On trouve en très grand nombre les 
banques et les assurances qui proposent des produits de placement ou des formules de prévoyance pour les obsèques.

Les organismes de voyage et les représentants de régions et de pays touristiques se partagent également les emplacements ainsi que diverses associations de loisirs (cours d'écriture, réalisation de son arbre généalogique, etc.). On y trouve également des stands de beauté, de technologies, de sport et de bénévolat. Mais on voit également çà et là des stands concernant l'aménagement du lieu de vie et la prise en charge de certaines déficiences (maladies cardiovasculaires, fuites urinaires, pertes de mobilité, etc.).

Alors que notre société est souvent qualifiée de "jeuniste», accusée de favoriser à outrance la jeunesse (Billé et Martz 2010), et que le marketing est suspecté de ne présenter qu'une image édulcorée du vieillissement, la question qui se pose est de savoir comment ces salons parviennent à mettre en présence une forme de vieillissement valorisée (le « vieillir jeune ») et un vieillissement qui est sans cesse repoussé et qui est rarement montré (la dépendance)?

Tout d'abord, on peut constater que l'espace du salon est divisé en plusieurs zones thématiques regroupant des exposants qui participent à un même thème. Celui de Bruxelles est par exemple partagé en trois pôles : «Vivre » ou « comment profiter de sa vie ?», où l'on voit les banques et les assurances, les organismes de pension et les agences de résidences secondaires. «Partager» ou « comment prendre soin de soi et de sa famille ? », où l'on trouve les associations et tout ce qui concerne la santé. Enfin, « Profiter » qui regroupe les différentes organisations destinées aux loisirs et au corps (voyage, beauté, mode et décoration). Ainsi, la façon dont sont conçus ces salons permet d'effacer en partie la « vieillesse-dépendance », de la rendre moins visible en la diluant dans la masse des produits et services présentés.

Ensuite, on remarque que les discours produits autour de cette vieillesse ne s'adressent pas directement aux visiteurs du salon, mais à la génération qui les précède : «Vos parents sont âgés et vous voulez les aider à bien vivre chez eux, en leur permettant de rester chez eux le plus longtemps possible ? Le village "bien vivre chez moi” vous apporte deux types de services »1. La dépendance est ainsi repoussée à un avenir qu'il est nécessaire de préparer, mais qui ne concerne pas encore les visiteurs. Le corps, quant à lui, est moins envisagé dans sa dimension médicale que dans une optique de prévoyance ou de bien-être, gommant la maladie au profit du bienvieillir : «Thalassothérapie, thermalisme, spa, médecines douces, compléments alimentaires, équipements et clubs sportifs, beauté, produits de bien-être... autant de façons de prendre soin de vous qui vous attendent au Salon des Seniors » 2.

1 Salon des Seniors, http://www.salondesseniors.com/le-salon/10-villages-thematiques. Consulté le 22 mai 2013

$2 \mathrm{lbid}$. 
Enfin, ces salons véhiculent l'image d'un vieillissement proactif. Zénith 50 par exemple (le salon de Bruxelles), organise un défilé de mode dont les mannequins n'ont guère plus de 63 ans, ainsi qu'un concours de Miss et Mister Senior dont le gagnant doit présenter les caractéristiques suivantes :

«Êtes-vous le plus actif des plus de 50 ans?

- courir un marathon tous les week-ends ;

- travailler comme volontaire à la Croix rouge ;

- être trésorier d'un mouvement féminin local ;

- s'occuper de ses petits-enfants tous les jours.

Bref, un senior actif est quelqu'un qui profite de la vie et qui aime les contacts sociaux $»^{3}$.

Globalement, ces salons participent donc à la promotion d'une image positive de la personne vieillissante en investissant davantage les jeunes retraités qui disposent de plus de moyens financiers. Ils n'excluent pas la dépendance, mais la gomment et la repoussent à un avenir lointain. Ils contribuent à la production d'un modèle de vieillesse fondé sur la consommation de loisirs, tout en mettant l'accent sur l'hygiène de vie, l'anticipation et le contrôle de son vieillissement. On voit donc le retraité actif, en bonne santé, prenant soin de son corps, profitant du temps disponible et préparant son futur (santé et mort) (Gilleard 2005). Autrement dit, ces salons promeuvent une résistance au vieillissement du corps et de l'esprit par une action individuelle (Feillet et al. 2010).

Si ces messages ne dictent pas directement les manières d'agir des personnes retraitées, ils diffusent toutefois un discours moral qui encadre les actions individuelles (Laliberte Rudman 2006), ainsi que des images auxquelles ils sont amenés à s'identifier. Nous allons voir que si la majorité des répondants résistent à l'image très homogène des seniors, ils ont davantage intégré les exigences de se prendre en main et d'être actif.

\section{Les résistances individuelles}

\subsection{Résistance au vieillissement physique, conformisme au marketing senior}

Avec la cessation de l'activité professionnelle, le corps prend davantage de place dans les préoccupations individuelles : la retraite libère un temps pour penser à son corps et les maux qui lui sont associés ne sont désormais plus attribués aux conditions de travail. Les activités quotidiennes amènent à la conscience de ce vieillissement corporel inéluctable: les performances sportives s'amoindrissent, s'occuper des petitsenfants fatigue, monter les escaliers devient plus difficile, etc. Le constat du vieillis-

3 Zénith 50, http://www.salonzenith.be/fr. Consulté le 13 novembre 2011. 
sement s'impose aux personnes retraitées qui cherchent à repousser ou à éviter le vieillissement de leur corps en mettant en place des stratégies de préservation de l'autonomie physique et intellectuelle.

La santé devient alors un capital qu'il est nécessaire de préserver et l'action personnelle en est le moyen privilégié. Bien conscients qu'une grande partie de ce capital est le fruit du hasard, il y a toutefois chez beaucoup de répondants une volonté de garder une certaine maîtrise de leur corps en mettant toutes les chances de leur côté.

Ils appliquent les conseils glanés dans des revues, chez le médecin, à la télévision, auprès de la famille ou des amis. Ces conseils sont fondés sur une rationalisation médicale : le nombre de pas qu'il faut effectuer chaque jour pour rester en bonne santé, les types de sport que l'on peut faire, les aliments que l'on doit manger ou au contraire ceux qui sont à proscrire, etc.

«Je fais l'effort de faire une demi-heure de marche tous les jours, donc je fais quand même un effort pour ma santé. Pas assez par rapport à ce que je devrais faire. Je devrais faire 10000 pas par jour m'a dit le médecin... On a un podomètre maintenant, un truc qu'on met, et je dois faire 10000 pas et je n'arrive qu'à 2500. II est dit que pour garder une bonne santé il faut faire 10 000 pas par jour. Et je regarde mon podomètre en fin de journée et je suis chaque fois très découragé » (Robert, 60 ans, manager).

Les répondants partagent également l'idée que les facultés intellectuelles peuvent être préservées par une action personnelle. C'est dans ce but que certains s'inscrivent à des cours de langues ou à l'université des aînés, qu'ils remplissent des grilles de mots croisés quotidiennement, etc.

Ce faisant ils luttent contre le spectre de la "vieillesse-dépendance » assimilée à une perte quasi totale d'autonomie physique et des capacités intellectuelles. La crainte de la dépendance est d'autant plus marquée dans les récits de personnes qui ont fait l'expérience de cette vieillesse, comme chez cet ancien infirmier qui a travaillé avec des personnes âgées mourantes, chez cette femme qui a accompagné sa mère octogénaire dans la maladie ou encore chez les personnes qui sont elles-mêmes passées par la maladie.

Tout en mettant en place des stratégies concrètes de résistance au vieillissement corporel, les répondants s'appliquent à ne pas se projeter dans cet avenir incertain. C'est par un travail mental de résistance qu'ils choisissent de vivre davantage dans le présent et de profiter du temps qu'il leur reste en bonne santé.

« $Q$ : Vous pensez parfois à préparer l'avenir?

- $\mathrm{P}$ : Se mettre en maison de retraite? non vraiment pas! On a déjà des activités jusqu'à la fin de l'année et chaque mois on ajoute à notre avenir donc ça suit » (Philippe, 65 ans, cadre).

\subsection{Ni jeunes ni vieux : résistance aux images de la vieillesse}

À côté des contraintes physiques liées au vieillissement du corps, les retraités opèrent une résistance à des contraintes morales de deux types : celles qui visent à assigner les 
personnes à une catégorie d'âge uniforme, et celles qui associent l'âge avancé à la vieillesse.

La première consiste à réduire la diversité des vécus individuels à une image homogène de la retraite. En présentant les «seniors » comme ayant les mêmes goûts, les mêmes loisirs, les mêmes valeurs, les mêmes modes de vie, le marketing senior opère une telle homogénéisation. Et nombre de répondants disent ne pas se reconnaître dans cette catégorie trop réductrice.

La résistance se formalise alors par une tension entre la conscience de l'âge comme marqueur objectif de vieillesse (« je sais que je suis vieux ») et le sentiment de ne pas se reconnaître dans la catégorie imaginaire des vieux, celle qui n’existe en réalité qu’à travers les représentations que l'on en fait et qui est relayée dans les médias :

«Mais on n'est pas abonné à ça encore (à une revue visant spécifiquement les plus de 50 ans), ça va peut-être venir. J'ai déjà lu des articles, je dois être honnête, j'ai déjà lu ça dans les salles d'attente. Mais en fait je pense que pour l'instant je ne suis pas du tout prête à rejoindre le bataillon des seniors comme ça, unigénérationnel » (Camille, 60 ans, employée administrative).

La seconde contrainte morale est opérée par l'entourage ou par la personne ellemême et consiste à associer la retraite et l'âge biologique à la vieillesse. Dans les récits, être vieux c'est «faire les courses avec bobonne » (Guy), « rester dans son fauteuil à végéter » (Alain), « être éteint » (Cécile), « avoir une attitude misérabiliste, n'avoir envie de rien »(Aline). La retraite est perçue par les répondants comme un risque majeur de basculement précoce dans cette vieillesse qu'ils redoutent.

«On en connaît des personnes qui ont pris leur pension comme ça et qui se sont un peu endormies. Ils n'ont pas commencé d'activités, ils n'ont pas continué à fréquenter du monde et puis ça se rétrécit tout le temps. On devient une plante finalement » (Marie-Laure, 58 ans, institutrice).

La tension naît du fait que les âges objectif et subjectif sont différenciés : si objectivement ils reconnaissent faire partie des seniors de par leur âge, les répondants ne se sentent subjectivement pas appartenir à cette catégorie figée à laquelle ils associent des représentations négatives :

"Je n'ai pas honte de dire mon âge, j'ai 61 ans et voilà. Je suis senior, mais je n'ai pas l'impression d'avoir besoin d'être coaché, ou d'avoir des idées ou... mais sinon je n'ai pas du tout envie de me sentir senior, d'être embrigadé dans le salon des seniors » (Guy, 63 ans, enseignant).

Cette tension engendre un travail mental de mise à distance de la catégorie en modifiant la manière de se voir, en s'affirmant en opposition à la catégorie des " seniors » ou des « vieux»:

«Un jour ma fille aînée m'a dit “mais maman, arrête un peu, assieds-toi, tu sais, tu deviens fragile" et je l'ai regardée "moi, je deviens fragile ?", mais c'était mignon hein, elle prenait soin de moi. Je lui ai dit "écoute chouchou je n'ai quand même pas encore les 80 berges hein" et on en a ri » (Marion, 64 ans, psychologue).

Comme l'a montré Vincent Caradec, la comparaison avec d'autres personnes âgées joue un rôle important dans ce travail de mise à distance (Caradec 2007). Les répon- 
dants se définissent bien souvent par rapport à des personnes de leur entourage qu'ils perçoivent comme « vieux»:

« Il y a des gens qui sont dans l'isolement le plus total ! Le frère d'Ivan, il veut plus sortir de chez lui, bon, il est comme ça depuis des décennies, c'est désastreux! C'est dans la tête qu'on devient vieux d'abord hein » (Aline, 64 ans, enseignante).

Pour résister à toutes ces assignations, les personnes interrogées mettent également en place des actions concrètes. Ils évitent par exemple les activités qui leur renvoient une image d'eux comme faisant partie des «vieux ». Il s'agit de favoriser des activités transgénérationnelles, en préférant suivre les cours à l'université avec les étudiants plutôt qu'avec les aînés ou d'éviter les loisirs qui renvoient à l'image qu'ils ont de la vieillesse :

«L'aquagym en soi ça a l'air bien, mais alors je vais peut-être prendre un bouquin ou bien prendre deux trois cours et puis le faire seule. Je ne me vois pas avec ces mémés, leur bonnet de bain à fleurs, enfin bon je ne me vois pas comme ça, pas encore (rires) ! »(Marion, 64 ans, psychologue).

Ils mettent également en place un travail - certains parlent d'effort à faire - pour garder des contacts, ne pas s'isoler et ne pas tomber dans l'oisiveté :

«Toutes mes occupations contribuent à ne pas tomber dans la déprime, parce que qui dit déprime dit inactif, qui dit inactif, l'organisme ne suit plus et c'est à ce moment-là que les petits problèmes commencent...Il y a pas mal de gens, je vois autour de moi, qui sont partis à la pension avant moi, et il n'y a plus d'occupation, j'ai des collègues qui sont déjà décédés... Parce qu'une fois qu'on part en pension on est dans un congé éternel, il ne faut plus se lever le matin, mais si justement ! II faut continuer à s'entretenir, à se motiver à se lever le matin... Donc je m'oblige à ne pas rester immobile dans un fauteuil, à me ronger et à dire "bon voilà j'ai 60 ans, qu'est-ce qu'il me reste ?" » (Gus, 64 ans, policier).

Il est important de souligner ici la récurrence de la notion de projet. Beaucoup de répondants insistent sur l'importance, au-delà des loisirs, d'avoir des projets, de s'investir dans des activités qui donnent un sens à la retraite.

«Mais pour le moment je ne sais pas encore très bien les activités bien définies "tiens je vais faire ça, je vais faire ça", on vit un petit peu au jour le jour. Finalement ça ne me dérange pas tellement, mais il paraît que ce n'est pas bon. L'entourage qui dit "attention il faut trouver des activités", des activités bien précises et avec horaires et avec certaines contraintes, parce que sinon tu es vite, bon on reste dans son fauteuil à végéter » (Alain, 65 ans, ingénieur).

La fonction de ces projets, aussi divers soient-ils, n'est pas uniquement d'apporter une occupation et de pallier l'ennui potentiel du temps long de la retraite. Ils permettent surtout une projection de soi dans des rôles, des activités, qui donnent un sens à l'existence. C'est grâce à eux que la personne se raconte, se reconnaît et est reconnue 
comme une personne par son entourage4. Le projet est donc un moyen d'opérer le travail mental de résistance contre l'association de la retraite à la vieillesse et de la vieillesse à l'oisiveté.

\section{Un modèle normatif excluant ?}

Le marketing senior prône une résistance au vieillissement par la proactivité, projetant le modèle d'une « retraite-épanouissement ». Nous avons détaillé la manière dont ce cadre normatif était globalement partagé par tous les répondants. Mais il n'embrasse pour autant pas toute la réalité sociale identifiée dans notre échantillon. En ne visant qu'une catégorie de consommateurs aisés, le marketing met sur la touche les retraités qui ne font pas partie de ces nouveaux consommateurs.

Dans deux des récits recueillis, les notions de résistance, de responsabilité individuelle et d'épanouissement personnel ne prennent pas la même forme. D'abord parce qu'elles ne sont pas évoquées explicitement dans les entretiens. Ensuite parce que les valeurs sur lesquelles ces répondants s'appuient ne sont pas les mêmes.

Il s'agit de deux hommes, anciens ouvriers, disposant de faibles revenus. Pour eux, la responsabilité d'un homme tient en ce qu'il s'occupe bien de son ménage. Sa valeur se mesure dès lors à sa capacité à travailler pour faire vivre sa famille.

«Beaucoup a tenu par la nécessité de faire bouillir la marmite. Donc je pense que c'était pour la famille, il fallait ne pas perdre son boulot, etc., donc ça a été pendant des années et des années mon but principal» (Georges, 65 ans, conducteur de bus).

Le corps vieillissant représente une limite à cette capacité : on n'est plus rien lorsqu'on ne peut plus travailler parce que le corps n'en est plus capable.

«-Q: vous aimeriez bien travailler jusque quand?

- M : Je sais pas, jusque je vis, jusque je sais encore marcher, je sais pas dire moi. Moi ma vie je sais plus rien dire. J'ai bien travaillé jusque 65 ans. Mais j'ai eu ça à cette épaule, ou sinon j'étais encore au travail. Mais à 65 ans on peut plus travailler. II y en a qui travaillent jusque 70 ans, comme le roi. Et pourtant il pouvait déjà avoir sa pension celui-là, le roi ! Et la reine ? Eux peuvent bien travailler, pourquoi nous autres on peut pas si on sait? 》 (Maurice, 65 ans, balayeur).

Dans ces récits, la dichotomie jeune/vieux n'est pas évoquée, le vieillissement n'est pas une question d'action personnelle, mais de contraintes extérieures et le passage à la retraite n'est pas inscrit dans un projet de réalisation de soi. La retraite devient alors la longue attente de la mort comme l'avait noté Anne-Marie Guillemard (Guillemard 1972) :

4 Sur la notion de projet et la construction de l'identité à la retraite, Eraly (2013), « L'identité narrative mise à l'épreuve de la retraite : une analyse de récits biographiques ». In Temporalités, 17/2013, article en ligne : http://temporalites.revues.org/2458. 
«À vous dire franchement, je m'emmerde. Oui, très clairement. En fait j'ai aussi l'impression d'être... Moi j'attends le stade suivant quoi » (Georges, 65 ans, conducteur de bus).

Passer à la retraite signifie arrêter de travailler et perdre sa valeur, celle qui fait que l'on existe en tant qu'homme. Les deux répondants souhaitent opposer une résistance à cette fatalité, en continuant à travailler jusqu'à ce que le corps ne le permette plus. Mais ils se heurtent à l'obligation légale d'arrêter leur activité professionnelle. Après quelques mois passés à la retraite, la seule issue possible se trouve alors dans la résignation et non dans l'épanouissement ou l'action.

\section{Conclusion}

L'objectif de cet article était d'analyser la (re)production d'une norme sociale, en partant de l'étude d'un canal de diffusion - le marketing senior - et en mettant en évidence les manières dont les individus se la réapproprient dans leurs pratiques et leurs discours. Il ne s'agissait pas de réduire notre compréhension du marketing à une idéologie qui s'imposerait du haut vers le bas, les multiples adaptations individuelles montrant bien au contraire que la relation entre les discours normatifs et les vécus individuels est plus complexe et qu'on ne peut les penser indépendamment.

Nous avons mis en évidence que le marketing senior invite à résister au vieillissement. Les moyens prônés pour y parvenir sont l'épanouissement personnel et l'action individuelle : rester maître de son vieillissement tout en profitant de sa retraite. Le marketing senior s'est fait le relais d'une rationalisation médicale de la retraite, il s'est engouffré dans ce mouvement, en proposant des moyens matériels à cette résistance (des produits anticholestérol, des outils d'entraînement cérébral, etc.). Mais en se faisant le relais du vieillissement actif, en valorisant l'action personnelle et l'épanouissement, il offre surtout les moyens moraux pour résister au vieillissement. Il est à la fois un moteur pour les actions, il motive les choix individuels, et il est le cadre moral à partir duquel les individus jugent leurs actes.

Les récits de jeunes retraités révèlent deux formes de résistance. La première concerne les contraintes physiques. Le passage à la retraite éveille la conscience du corps qui vieillit et agite la peur de la dépendance, et les répondants s'attachent à minimiser les effets d'une contrainte qui leur semble quasiment inévitable. La seconde forme de résistance est opérée par rapport à des contraintes morales. Les jeunes retraités ne souhaitent pas se laisser assigner par leur entourage, par les médias ou par leurs propres représentations à des catégories homogènes telles que «les seniors » ou " les vieux».

Nous avons mis en évidence deux moyens de résistance par les individus à ces contraintes. C'est d'une part par des actions concrètes que ceux-ci repoussent le moment de la vieillesse et se détachent de toute catégorisation. Nous avons évoqué l'action sur le corps et la mise en place de divers projets qui permettent de ne pas s'isoler. D'autre part, les retraités opèrent un travail mental de mise à distance par rapport aux caté- 
gories homogénéisant l'avancée en âge. Nous avons vu qu'ils dissociaient leur âge objectif et leur âge subjectif en se comparant aux autres. Nous avons également évoqué le fait que grâce aux projets qu'ils mettent en place, ils s'offrent la possibilité de se raconter à partir d'éléments concrets et de se projeter dans de nouveaux rôles. C'est enfin en ne pensant pas à l'avenir, en cherchant à s'épanouir dans le présent, qu'ils résistent à la vieillesse et aux représentations négatives qu'ils lui associent.

Il était enfin nécessaire de souligner le fait que ce modèle de "retraiteépanouissement » est largement dominant dans les représentations collectives et très certainement bien présent dans la réalité des vécus individuels. Mais il laisse dans l'ombre tous ceux qui ne partagent pas les valeurs modernes de réalisation de soi et de responsabilité individuelle, ou tout du moins qui ne les intègrent pas de la même manière dans leurs vécus et dans leurs récits. Pour ceux-là, le corps prend une importance différente, il n'est pas envisagé comme un capital qu'on doit entretenir, il reste davantage l'outil de travail, celui qui fonde le rôle d'un individu et donc, sa valeur. Si l'action concrète est un moyen de résistance au vieillissement (en continuant à travailler), elle se heurte à une impossibilité légale (après 65 ans on ne peut plus travailler) et la résignation semble être alors la seule issue possible.

\section{Bibliographie}

Antoine J. (2003) : «Un très bon public “cible” pour le marketing ». Gérontologie et société, vol. 3 n¹06, pp. 279-289.

Baudrillard J. (1970) : La société de consommation. Paris : Éditions Denoël.

Billé M., Martz D. (2010): La tyrannie du « bien vieillir ». Lormont : Le bord de l'eau.

Bradley D.E., Longine C.F.J. (2001): « How Older Poeple Think About Images of Aging in Advertising and the Media ». Generations, vol. $25 \mathrm{n}^{\circ} 3$, pp. 17-21.

Caradec V. (2005): “"Seniors” et "personnes âgées”. Réflexions sur les modes de catégorisation de la vieillesse ». Cahiers d'histoire de la sécurité sociale, n¹, pp. 313326.

Caradec V. (2007): « L'épreuve du grand âge ». Retraite et Société, n52, pp. 12-37.

Cousin S. (2005): « Exposer le tourisme culturel au salon ». Ethnologie française, vol. 35 $\mathrm{n}^{\circ} 1, \mathrm{pp} .55-62$.

Dacheux É. (2001): «Étudier le marketing à la lumière de la communication ». L'année sociologique, vol. $51 \mathrm{n}^{\circ} 2$, pp. 411-427.

Desjeux D. (2010): " Anthropologie de la consommation et sociologie du consumérisme ». Cours de sociologie de la consommation. 
Eraly H. (2013): « L’identité narrative mise à l'épreuve de la retraite : une analyse de récits biographiques ». Temporalités, 17/2013, article en ligne : http://temporalites.revues.org/2458.

Feillet R., Bodin D., Héas S. (2010): « Corps âgé et médias: entre espoir de vieillir jeune et menace de la dépendance ». Études de communication, n³5, pp. 149-166.

Gestin A. (2001): «Un nouvel impératif pour les hommes et les femmes retraités: "vieillir jeune” ». Cahiers du Genre, n³1, pp. 203-219.

Gestin A. (2003): « Temps, espaces et corps à la retraite: des paradoxes à penser ». L'homme et la société, n¹47, pp.169-190.

Giddens A. (1991): Modernity and Self-Identity. Cambridge: Polity Press.

Gilleard C. (2005): "Cultural approaches to the Ageing body ». In The Cambridge Handbook of Age and Ageing, edited by Johnson M.L. Cambridge: Cambridge University Press, pp.156-164.

Gilleard C., Higgs P. (2011) : "Consumption and Aging ». In Handbook of Sociology of Aging, edited by Settersten J.R.A., Angel J.L. New York: Springer, pp. 361-375.

Guillemard A.-M. (1972) : La retraite, une mort sociale. Sociologie des conduites en situation de retraite. Paris: Mouton.

Hénaff-Pineau P.-C. (2009): « Vieillissement et pratiques sportives : entre modération et intensification ». Lien social et politique, $n^{\circ} 62$, pp. 71-83.

Hewitt A. (2010): « Retirement: What will you do? A narrative inquiry of occupationbased planning for retirement: Implications for practice ». Australian Occupational Therapy Journal, n57, pp. 8-16.

Honneth A. (2006): La société du mépris. Vers une nouvelle théorie critique. Paris: La Découverte.

Laliberte Rudman D. (2006): "Shaping the active, autonomous and responsible modern retiree : an analysis of discursive technologies and their links with neoliberal political rationality ». Ageing of Society, n²6, pp. 181-201.

Martuccelli D. (2002): Grammaires de l'individu. Paris : Gallimard.

Moulaert T., Léonard D. (2011): « Le vieillissement actif sur la scène européenne ». Courrier hebdomadaire du CRISP, vol. 20 n²105, pp. 5-33.

Ricoeur P. (1990): Soi-même comme un autre. Paris : Seuil.

Ruaux J.-Y. (2007): « Vieux, seniors, aînés... Cible des médias et médias eux-mêmes ». Gérontologie et société, vol. 1 n¹20, pp. 77-90. 
Walker A. (2009): « Commentary: The Emergence and Application of Active Aging in Europe ». Journal of Aging e Social Policy, vol. 1 n²1, pp. 75-93. 